\title{
Stability Analysis of Fractional Order Memristor Synapse-coupled Hopfield Neural Network with Ring Structure
}

\section{Leila Eftekhari}

Tarbiat Modares University

Mohammad Amirian ( $\square$ M.amirianmatlob@dal.ca)

Dalhousie University https://orcid.org/0000-0002-8402-5779

\section{Research Article}

Keywords: tional Calculus, Bifurcation, Stability, Memristor, Hopfield Neural Network

Posted Date: October 19th, 2021

DOl: https://doi.org/10.21203/rs.3.rs-973554/v1

License: (c) (1) This work is licensed under a Creative Commons Attribution 4.0 International License.

Read Full License 


\title{
Stability Analysis of Fractional Order Memristor Synapse-coupled Hopfield Neural Network with Ring Structure
}

\author{
Leila Eftekharia ${ }^{\mathrm{a}}$ Mohammad M. Amirian ${ }^{\mathrm{b}, *}$ \\ ${ }^{a}$ Department of Mathematics, Tarbiat Modares University, Tehran, IR 14117-13116 \\ ${ }^{b}$ Department of Mathematics and Statistics, Dalhousie University, Halifax, NS, CA B3H4R2
}

\begin{abstract}
A memristor is a non-linear two-terminal electrical element that incorporates memory features and nanoscale properties, enabling us to design very high-density artificial neural networks. To examine the embedded memory property, we should use mathematical frameworks like fractional calculus, which is capable of doing so. Here, we first present a fractional-order memristor synapse-coupling Hopfield neural network on two neurons and then extend the model to a neural network with a ring structure that consists of $n$ sub-network neurons. Necessary and sufficient conditions for the stability of equilibrium points are investigated, highlighting the dependency of the stability on the fractional-order value and the number of neurons. Numerical simulations and bifurcation analysis, along with Lyapunov exponents, are given in the two-neuron case that substantiates the theoretical findings, suggesting possible routes towards chaos when the fractional order of the system increases. In the $n$-neuron case also, it is revealed that the stability depends on the structure and number of sub-networks.
\end{abstract}

Keywords: Fractional Calculus, Bifurcation, Stability, Memristor, Hopfield Neural Network

\section{Introduction}

The brain as the central organ of the nervous system regulates most of the activities, such as processing, integrating, etc in our body. Brain dynamics is generally studied based on mathematical equations, more specifically neural networks models [1]. Among these models, Hopfield neural network draws considerable attention due to its simple paradigm [2,3]. Using memristors instead of resistors in the Hopfield neural network models, one can build a new system where the parameters vary according to its state, which is said to be a memristor-based neural network. The memory features and nanoscale properties of the memristor enable us to design very high-density systems. It is shown that memristors can be used as synaptic weights in artificial neural networks due to their learning ability [4]. To examine the behavior of such complex systems, scholars should use non-linear-based models, like fractional calculus. For instance, in 2020, Aguilar et al used a fractionalized version of neural networks to study system identification, and they have found that their model has a higher precision with a fewer number of parameters compared to integer models [5].

Fractional calculus (FC) is a generalised version of integer calculus and a specific case of convolution integral. Unlike ordinary calculus, definitions in FC are not unique, and we have various operators under different weight functions, giving us a wide range of opportunity to study complex systems incorporating memory properties [6-10]. However, some basic theorems and algebra lemmas such as chain rule and

Email address: M.Amiriammatlob@dal.ca, ORCID: 0000-0002-8402-5779 (Mohammad M. Amirian) 
stability criteria holding for integer calculus is no longer valid when it comes to Fc, so careful attention is required when we model complex systems with FC.

This paper aims to build up solid mathematical frameworks for the stability criteria of n-dimensional fractional-order network models. To do so, we first introduce our fractional-order memristor synapsecoupled Hop-field neural network (FMHNN) on two neurons using fractional derivative in sense of Caputo (Eq. 4). We chose this fractional operator since it only requires initial conditions given in terms of integerorder derivatives [11], consisting with the property of our model. Applying the ring network concept on equations, we then expand our model to a complex system, where each membrane is coupled by $n$ subnetworks. After building the required fundamental theoretical bases for stability analysis, we examine the criteria numerically for both systems. To study the finding in more detail, we also investigate the impact of the initial condition and fractional order on FMHNN model through performing bifurcation analysis.

\section{Method and Material}

\subsection{Models}

FMHNN Model. Using the definition (2.1), we extend the concept of the integer-order memristor synapse-coupled Hopfield neural network (MHNN), represented in 2019 [12], to FMHNN in the following form

$$
\left\{\begin{array}{l}
{ }_{0}^{\mathrm{c}} D_{t}^{\alpha} x_{1}=-x_{1}+b_{1} \tanh \left(x_{1}\right)+b_{2} \tanh \left(x_{2}\right)+k \varphi\left(x_{1}-x_{2}\right), \\
{ }_{0}^{\mathrm{c}} D_{t}^{\alpha} x_{2}=-x_{2}+b_{3} \tanh \left(x_{1}\right)+b_{4} \tanh \left(x_{2}\right)-k \varphi\left(x_{1}-x_{2}\right), \\
{ }_{0}^{\mathrm{c}} D_{t}^{\alpha} \varphi=x_{1}-x_{2} .
\end{array}\right.
$$

where $0<\alpha \leq 1$ is the fractional order, and $b_{i}$ are constant parameters. Note that the FMHNN model reduces to MHNN model when $\alpha=1$.

In this model, when there is a potential difference between the neurons, an induction current will appear, and a two-way induction current $I_{M}$ flows through an active flux controlled memristor synapse as shown below

$$
\left\{\begin{array}{l}
I_{M}=W(\varphi) V_{M}=k \varphi V_{M} \\
\varphi=f\left(V_{M}\right)=V_{M}
\end{array}\right.
$$

where $\varphi$ and $V_{M}$ account for the state variable of the memristor, and the potential difference of the two neurons, respectively; $W=k \varphi$ also explains a memductance function with memristor coupling weight $k$. $x_{1}$ and $x_{2}$ are the state variables describing the potentials between the inside and outside of the two neurons, $\tanh \left(x_{1}\right)$ and $\tanh \left(x_{2}\right)$ are the activation function of the two neurons, and $V_{M}=x_{1}-x_{2}$ is the potential difference of the two neurons. The coefficients represent the amplitude of a connection between two neurons. The FMHNN schematic connection pattern of two neurons is shown in figure (1).

FMHNN Model with Ring Structure. We apply a ring-network structure on FMHNN model (1), and by doing so we expand our previous model from two neurons to $n$ units of neurons. The ring network consists of $n$ sub-networks coupling strength among all membrane potentials $x_{1 i}$ in the following form

$$
\left\{\begin{array}{l}
{ }_{0}^{\mathrm{c}} D_{t}^{\alpha} x_{1 i}=-x_{1 i}+b_{1} \tanh \left(x_{1 i}\right)+b_{2} \tanh \left(x_{2 i}\right)+k \varphi_{i}\left(x_{1 i}-x_{2 i}\right)+\frac{d}{2 p} \sum_{j=i-p}^{i+p}\left(x_{1 j}-x_{1 i}\right), \\
{ }_{0}^{\mathrm{c}} D_{t}^{\alpha} x_{2 i}=-x_{2 i}+b_{3} \tanh \left(x_{1 i}\right)+b_{4} \tanh \left(x_{2 i}\right)-k \varphi_{i}\left(x_{1 i}-x_{2 i}\right), \\
{ }_{0}^{\mathrm{c}} D_{t}^{\alpha} \varphi_{i}=x_{1 i}-x_{2 i} .
\end{array}\right.
$$




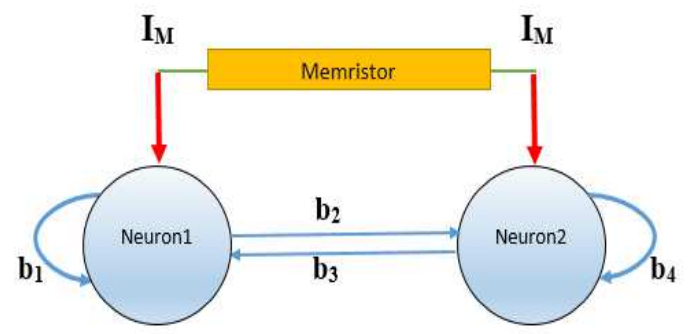

Figure 1: The FMHNN connection pattern with two neurons.

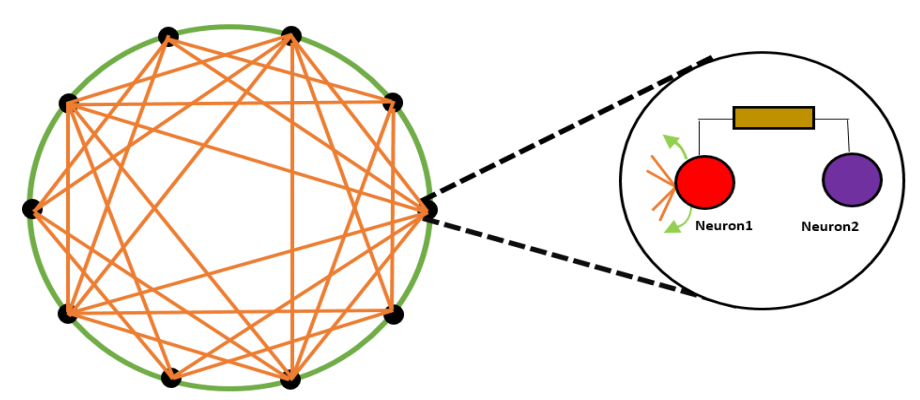

Figure 2: The FMHNN connection pattern of $n=10$ sub-networks of neurons with a ring structure where $P=2$.

where $i=1,2, \ldots, n$, and $\left[x_{1 i}, x_{2 i}, \varphi_{i}\right]$ represent the state variables of $i^{\text {th }}$ sub-network. Futher, the memristor synapses are coupled symmetrically to their $2 P$ nearest neighbourhood by neural sub-networks, interacting with $P$ neighbourhood from of left and right side (Fig. 2). Also, $d$ stands for the coupling strength among the $n$ sub-networks.

Definition 2.1. [13] For a continuous function $f$, with $f^{\prime} \in L^{1}\left(R^{+}\right)$, the Caputo fractional-order derivative of order $\alpha \in(0,1)$ is defined by

$$
{ }_{t_{0}}^{\mathrm{c}} D_{t}^{\alpha} f(t)=\frac{1}{\Gamma(1-\alpha)} \int_{t_{0}}^{t}(t-s)^{-\alpha} f^{\prime}(s) d s .
$$

\subsection{Model Analysis}

Here, we study the existence and uniqueness of solutions and determine the stability criteria for both models $(1 \& 3)$. To do so, some fundamental definitions are required; each subsection therefore starts with those information. 
2.2.1. Existence and Uniqueness of Solutions

Definition 2.2. The circulant matrix is a square matrix in which each row has the previous row components shifted cyclically one place towards the right side.

$$
B=\operatorname{circ}\left(b_{0}, b_{1}, \ldots, b_{n-1}\right)=\left[\begin{array}{ccccccc}
b_{0} & b_{1} & b_{2} & b_{3} & \ddots & b_{n-2} & b_{n-1} \\
b_{n-1} & b_{0} & b_{1} & b_{2} & \ddots & b_{n-3} & b_{n-2} \\
b_{n-2} & b_{0} & b_{1} & b_{2} & \ddots & b_{n-4} & b_{n-3} \\
\ddots & \ddots & \ddots & \ddots & \ddots & \ddots & \ddots \\
b_{2} & b_{3} & b_{4} & b_{5} & \ddots & b_{0} & b_{1} \\
b_{1} & b_{2} & b_{3} & b_{4} & \ddots & b_{n-1} & b_{0}
\end{array}\right]_{n \times n}
$$

Also, the block circulant matrix is the generalized version of the above matrix where each component consist of the circulant matrix

$$
B=\operatorname{bcirc}\left(B_{0}, B_{1}, \ldots, B_{n-1}\right)=\left[\begin{array}{ccccccc}
B_{0} & B_{1} & B_{2} & B_{3} & \ddots & B_{n-2} & B_{n-1} \\
B_{n-1} & B_{0} & B_{1} & B_{2} & \ddots & B_{n-3} & B_{n-2} \\
B_{n-2} & B_{0} & B_{1} & B_{2} & \ddots & B_{n-4} & B_{n-3} \\
\ddots & \ddots & \ddots & \ddots & \ddots & \ddots & \ddots \\
B_{2} & B_{3} & B_{4} & B_{5} & \ddots & B_{0} & b_{1} \\
B_{1} & B_{2} & B_{3} & B_{4} & \ddots & B_{n-1} & B_{0}
\end{array}\right]_{n \times n}
$$

which $B_{i}($ for $i=0, \cdots, n-1)$ is a block matrices $[14,15]$.

Theorem 2.1. Assume that $\Omega=\left\{\left(x_{1}, x_{2}, \varphi\right) \in R^{3}: \max \{\|\varphi\|\} \leq \lambda\right\}$ and $\mathrm{S}=\Omega \times\left[t_{0}, T\right]$ where $T<\infty$. For any initial conditions $\left(x_{1}\left(t_{0}\right), x_{2}\left(t_{0}\right), \varphi\left(t_{0}\right)\right) \in \Omega$, all the solutions $\left(x_{1}(t), x_{2}(t), \varphi(t)\right) \in \mathrm{S}$ of system $(1)$ are unique for all $t \geq 0$.

Proof. Let $\left.\mathbb{F}(\mathrm{x})=\left(\mathbb{F}_{1}(\mathrm{x}), \mathbb{F}_{2}(\mathrm{x}), \mathbb{F}_{3}(\mathrm{x})\right)\right)^{T}$ be a mapping function with $\|$.$\| norm so that$

$$
\begin{aligned}
& \mathbb{F}_{1}(\mathrm{x})=-x_{1}+b_{1} \tanh \left(x_{1}\right)+b_{2} \tanh \left(x_{2}\right)+k \varphi\left(x_{1}-x_{2}\right), \\
& \mathbb{F}_{2}(\mathrm{x})=-x_{2}+b_{3} \tanh \left(x_{1}\right)+b_{4} \tanh \left(x_{2}\right)-k \varphi\left(x_{1}-x_{2}\right), \\
& \mathbb{F}_{3}(\mathrm{x})=x_{1}-x_{2}
\end{aligned}
$$

where $\mathrm{x}=\left(x_{1}, x_{2}, \varphi\right)$. Therefore, we can re-write our mapping function in a multi-variable form, $\mathbb{F}(\mathrm{x})=$ $A \mathrm{x}+B \tanh (\mathrm{x})+\varphi K \mathrm{x}$, such that

$$
A=\left[\begin{array}{ccc}
-1 & 0 & 0 \\
0 & -1 & 0 \\
1 & -1 & 0
\end{array}\right], \quad B=\left[\begin{array}{ccc}
b_{1} & b_{2} & 0 \\
b_{3} & b_{4} & 0 \\
0 & 0 & 0
\end{array}\right], \quad K=\left[\begin{array}{ccc}
k & -k & 0 \\
-k & k & 0 \\
0 & 0 & 0
\end{array}\right], \quad \mathrm{x}=\left[\begin{array}{c}
x_{1} \\
x_{2} \\
\varphi
\end{array}\right]
$$

We now prove that the system (1) satisfies the locally Lipschitz condition [8], i.e.

$$
\forall \mathrm{x}, \overline{\mathrm{x}} \in \mathrm{S}, \exists L \geq 0 \text { s.t }\|\mathbb{F}(\mathrm{x})-\mathbb{F}(\overline{\mathrm{x}})\| \leq L\|\mathrm{x}-\overline{\mathrm{x}}\|
$$


where $\mathrm{x}=\left(x_{1}, x_{2}, \varphi\right)^{T}$ and $\overline{\mathrm{x}}=\left(\overline{x_{1}}, \overline{x_{2}}, \bar{\varphi}\right)^{T}$.

$$
\begin{aligned}
\|\mathbb{F}(\mathrm{x})-\mathbb{F}(\overline{\mathrm{x}})\| & =\|A(\mathrm{x}-\overline{\mathrm{x}})+B(\tanh (\mathrm{x})-\tanh (\overline{\mathrm{x}}))+K(\varphi \mathrm{x}-\bar{\varphi} \overline{\mathrm{x}})\| \\
& \leq\|A\|\|\mathrm{x}-\overline{\mathrm{x}}\|+\|B\|\|\tanh (\mathrm{x})-\tanh (\overline{\mathrm{x}})\|+\|K \varphi\|\|\mathrm{x}-\overline{\mathrm{x}}\| \\
& \leq(\|A\|+\|B\|+\|K\|\|\varphi\|)\|\mathrm{x}-\overline{\mathrm{x}}\| \\
& \leq(\|A\|+\|B\|+\lambda\|K\|)\|\mathrm{x}-\overline{\mathrm{x}}\| \\
& =L\|\mathrm{x}-\overline{\mathrm{x}}\|
\end{aligned}
$$

where $L=(\|A\|+\|B\|+\lambda\|K\|)>0$. This means that with an initial condition $\mathrm{x}\left(t_{0}\right)=\left(x_{1}\left(t_{0}\right), x_{2}\left(t_{0}\right), \varphi\left(t_{0}\right)\right)^{T}$, FMHNN model (1) has an unique solution $\mathrm{x}(t) \in \mathrm{S}$.

Theorem 2.2. Assume that $\Omega=\left\{\left(\mathrm{x}_{1}, \mathrm{x}_{2}, \phi\right) \in \mathbb{R}^{n \times 3}\right.$, s.t $\mathrm{x}_{1}=\left[x_{1 i}\right]_{n \times 1}, \mathrm{x}_{2}=\left[x_{2 i}\right]_{n \times 1}, \phi=\left[\varphi_{i}\right]_{n \times 1}, \Lambda=$ $\left.\left[\lambda_{i}\right]_{n \times 1}, \max \{\|\phi\|\} \leq \Lambda\right\}_{i=1,2, \ldots, n}$ and also $S=\Omega \times\left[\mathrm{T}_{0}, \mathrm{~T}\right]$ where $\mathrm{T}=\left[T_{i}\right]_{n \times 1}, \mathrm{~T}_{0}=\left[t_{0 i}\right]_{n \times 1}$ for $i=1,2, \ldots$, n and $\mathrm{T}<\infty$. For any initial conditions $\left(\mathrm{x}_{1}\left(\mathrm{~T}_{0}\right), \mathrm{x}_{2}\left(\mathrm{~T}_{0}\right), \phi\left(\mathrm{T}_{0}\right)\right) \in \Omega$, all the solutions $\left(\mathrm{x}_{1}(\mathrm{~T}), \mathrm{x}_{2}(\mathrm{~T}), \phi(\mathrm{T})\right) \in S$ of system (3) are unique for all $\mathrm{T} \geq 0$.

Proof. Let $\mathbb{F}(\mathrm{X})=\left(\mathbb{F}_{1}(\mathrm{X}), \mathbb{F}_{2}(\mathrm{X}), \mathbb{F}_{3}(\mathrm{X})\right)^{T}$ be a multi-variable mapping function with $\|$.$\| norm so that$

$$
\begin{aligned}
& \mathbb{F}_{1}(X)=-\mathrm{x}_{1}+b_{1} \tanh \left(\mathrm{x}_{1}\right)+b_{2} \tanh \left(\mathrm{x}_{2}\right)+k \varphi\left(\mathrm{x}_{1}-\mathrm{x}_{2}\right)+\frac{d}{2 p} \sum_{j=i-p}^{i+p}\left(\mathrm{x}_{1 j}-\mathrm{x}_{1 i}\right), \\
& \mathbb{F}_{2}(X)=-\mathrm{x}_{2}+b_{3} \tanh \left(\mathrm{x}_{1}\right)+b_{4} \tanh \left(\mathrm{x}_{2}\right)-k \varphi\left(\mathrm{x}_{1}-\mathrm{x}_{2}\right), \\
& \mathbb{F}_{3}(X)=\mathrm{x}_{1}-\mathrm{x}_{2} .
\end{aligned}
$$

Therefore, we can re-write our mapping function in the following form

$$
\mathbb{F}(\mathrm{X})=A X+B_{1} \tanh (\mathrm{X})+B_{2} \tanh (\mathrm{X})+\varphi K \mathrm{X},
$$

where $A=\operatorname{bcirc}\left(A_{0}, A_{1}, \ldots, A_{1}\right) \in \mathbb{R}^{n \times n}, B_{1}=\left[\beta_{1}\right]_{1 \times n}, B_{2}=\left[\beta_{2}\right]_{1 \times n}, \beta_{1}=\left[b_{1}, b_{3}, 0\right], \beta_{2}=\left[b_{2}, b_{4}, 0\right]$, and $K=$ $\operatorname{bcirc}(\kappa, 0, . ., 0)_{1 \times n}$ so that $A_{0}, A_{1} \in \mathbb{R}^{3 \times 3}$ and

$$
A_{0}=\left[\begin{array}{ccc}
-1-\frac{d}{p} & 0 & 0 \\
0 & -1 & 0 \\
1 & -1 & 0
\end{array}\right], \quad A_{1}=\left[\begin{array}{ccc}
\frac{d}{2 p} & 0 & 0 \\
0 & 0 & 0 \\
0 & 0 & 0
\end{array}\right], \quad \kappa=\left[\begin{array}{ccc}
-k & k & 0 \\
k & -k & 0 \\
0 & 0 & 0
\end{array}\right]
$$

We now prove that the system (3) satisfies the locally Lipschitz condition [8], i.e.

$$
\begin{aligned}
& \forall \mathrm{X}, \overline{\mathrm{X}} \in \mathrm{S}, \exists L \geq 0 \text { s.t }\|\mathbb{F}(\mathrm{X})-\mathbb{F}(\overline{\mathrm{X}})\| \leq L\|\mathrm{X}-\overline{\mathrm{X}}\|, \\
\|\mathbb{F}(\mathrm{X})-\mathbb{F}(\overline{\mathrm{X}})\| & =\left\|A(\mathrm{X}-\overline{\mathrm{X}})+\left(B_{1}+B_{2}\right)(\tanh (\mathrm{X})-\tanh (\overline{\mathrm{X}}))+K(\varphi \mathrm{X}-\bar{\varphi} \overline{\mathrm{X}})\right\| \\
& \leq\|A\|\|\mathrm{X}-\overline{\mathrm{X}}\|+\left\|B_{1}+B_{2}\right\|\|\tanh (\mathrm{X})-\tanh (\overline{\mathrm{X}})\|+\|\Lambda\|\|K\|\|\mathrm{X}-\overline{\mathrm{X}}\| \\
& \leq\left(\|A\|+\left\|B_{1}\right\|+\left\|B_{2}\right\|+\|\Lambda\|\|K\|\right)\|\mathrm{X}-\overline{\mathrm{X}}\| \\
& =L\|\mathrm{X}-\overline{\mathrm{X}}\|,
\end{aligned}
$$

where $L=\left(\|A\|+\left\|B_{1}\right\|+\left\|B_{2}\right\|+\|\Lambda\|\|K\|\right)$. This means that with an initial condition $\mathrm{X}\left(t_{0}\right)=\left(\mathrm{x}_{1}\left(\mathrm{~T}_{0}\right), \mathrm{x}_{2}\left(\mathrm{~T}_{0}\right), \phi\left(\mathrm{T}_{0}\right)\right)^{T}$, FMHNN model with ring structure (3) has an unique solution $\mathrm{X}(\mathrm{T}) \in \mathrm{S}$. 


\subsubsection{Stability of Solutions}

Here, we build up the theoretical framework for the stability analysis of our models.

Definition 2.3. [6] For $0<\alpha<1$, the Laplace transform of Caputo fractional derivative, ${ }_{t_{0}}^{\mathrm{c}} D_{t}^{\alpha} f(t)$, derives from the following equation

$$
\int_{0}^{\infty} e^{-s t}{ }_{t_{0}} D_{t}^{\alpha} f(t) d t=s^{\alpha} F(s)-s^{\alpha-1} f\left(t_{0}\right)
$$

Definition 2.4. [6] For matrix $A \in R^{n \times n}$, the matrix Mittag-Leffler function is defined by

$$
E_{\alpha, \beta}[A]=\sum_{k=0}^{\infty} \frac{A^{k}}{\Gamma(\alpha k+\beta)}
$$

Lemma 2.1. [16] The linear autonomous system ${ }_{t_{0}}^{\mathrm{c}} D_{t}^{\alpha} \mathrm{x}=A \mathrm{x}$ is asymptotically stable if and only if

$$
|\arg (\lambda)|>\frac{\alpha \pi}{2} \text { when } \lambda \in \sigma(A)
$$

where $\sigma(A)$ denotes the spectrum of the matrix $A \in R^{n \times n}$.

Lemma 2.2. [17] The roots of the second order polynomial $p(\lambda)=\lambda^{2}+a \lambda+c$ satisfies inequality (6) if and only if $c>0$ and $b / \sqrt{c}<2 \cos (\alpha \pi / 2)$.

Lemma 2.3. [11] If $A \in R^{n \times n}, \beta$ is an arbitrary real number, and $\frac{\alpha \pi}{2}<\mu<\min \{\pi, \alpha \pi\}$, then a real positive constant, $r>0$, exists so that

$$
\left\|E_{\alpha, \beta}[A]\right\| \leqslant \frac{r}{1+\|A\|}, \quad \quad \mu \leq\left|\arg \left(\lambda_{i}[A]\right)\right| \leq \pi, \text { for } i=1,2, \ldots, n
$$

where $\lambda_{i}(A)$ denotes the eigenvalues of matrix $A$.

Lemma 2.4. [18] Assume that $u(t)$ and $f(t)$ are real-valued piecewise-continuous functions defined on the real interval $[a, b], K(t)$ is also real-valued and $K(t) \in L(a, b)$ and $u(t), K(t)$ are non-negative on this interval.

$$
\forall t \in[a, b], \quad u(t) \leqslant f(t)+\int_{a}^{t} K(\tau) u(\tau) d \tau
$$

and

$$
u(t) \leqslant f(t)+\int_{a}^{t} f(\tau) K(\tau) u(\tau) \exp \left\{\int_{\tau}^{t} K(s) d s\right\} d \tau .
$$

Theorem 2.3. Assume that we are given the following fractional order system with non-zero initial condition, $\mathrm{x}\left(t_{0}\right)=\mathrm{x}_{0}$ :

$$
{ }_{t_{0}}^{\mathrm{c}} D_{t}^{\alpha} \mathrm{x}(t)=A \mathrm{x}(t)+\mathbb{H}[\mathrm{x}(t)]
$$

where $0<\alpha \leq 1, A \in R^{n \times n}$ is a constant matrix, $\mathrm{x}=\left(x_{1}, x_{2}, \ldots, x_{n}\right)^{T} \in R^{n \times 1}$ is the state vector of our fractional-order system, ${ }_{t_{0}}^{\mathrm{c}} D_{t}^{\alpha} \mathrm{x}(t)$, which consists of the linear $A \mathrm{x}(t)$ and nonlinear $\mathbb{H}[\mathrm{x}(t)]$ parts. System (8) is locally asymptotically stable at its equilibrium point, $\mathrm{x}^{\star}$, when

$$
\left|\arg \left(\lambda_{i}\right)\right|>\alpha \frac{\pi}{2} \text {, and } \lim _{\mathrm{x} \rightarrow \mathrm{x}^{\star}} \frac{\|\mathbb{H}[\mathrm{x}(t)]\|}{\left\|\mathrm{x}-\mathrm{x}^{\star}\right\|}=0
$$

where $\lambda_{i}(i=1,2, . ., n)$ is the eigenvalues of matrix $A$. 
Proof. The equation (8) can be written in the following form after taking Laplace transform from it.

$$
s^{\alpha} \mathrm{X}(s)-s^{\alpha-1} \mathrm{x}_{0}=A \mathrm{X}(s)+L\{\mathbb{H}[\mathrm{x}(t)]\}
$$

therefore

$$
\mathrm{X}(s)=\left(s^{\alpha} I-A\right)^{-1}\left[s^{\alpha-1} X_{0}+L\{\mathbb{H}[(\mathrm{x}(t)]\}],\right.
$$

where $I$ is an $n \times n$ identity matrix. After taking Laplace inverse transform from the above equation (table $\mathrm{C} 1$ of [6]), we would have

$$
\mathrm{x}(t)=E_{\alpha, 1}\left(A t^{\alpha}\right) \mathrm{x}_{0}+\int_{0}^{t}(t-\tau)^{\alpha-1} E_{\alpha, \alpha}\left(A(t-\tau)^{\alpha}\right) \mathbb{H}[\mathrm{x}(\tau)] d \tau,
$$

Let $\mathrm{x}^{\star}$ be the solution of system (8) and $\lim _{\mathrm{x} \rightarrow \mathrm{x}^{\star}} \frac{\|\mathbb{H}[\mathrm{x}(t)]\|}{\left\|\mathrm{x}-\mathrm{x}^{\star}\right\|}=0$. Therefore

$$
\forall \varepsilon>0 \exists \delta_{0}>0 \text { s.t }\left\|\mathrm{x}(t)-\mathrm{x}^{\star}\right\|<\delta_{0} \Rightarrow\|\mathbb{H}[\mathrm{x}(t)]\|<\varepsilon\left\|\mathrm{x}-\mathrm{x}^{\star}\right\|
$$

Now let $\delta$ be chosen arbitrarily subject to $0<\delta<\delta_{0}$ and consider solutions for which $\left\|\mathrm{x}_{0}\right\|<\delta$. Form lemma (2.3)

$$
\exists r_{0}, r \in R^{+} \text {s.t }\left\|\mathrm{x}(t)-\mathrm{x}^{\star}\right\| \leqslant \frac{r_{0}\left\|\mathrm{x}_{0}\right\|}{1+\|A\| t^{\alpha}}+\int_{0}^{t} \frac{\varepsilon r_{0} r(t-\tau)^{\alpha-1}}{\left(1+\|A\|(t-\tau)^{\alpha}\right)}\left\|\mathrm{x}(\tau)-\mathrm{x}^{*}\right\| d \tau,
$$

Using Grönwall's inequality Lemma (2.4) and letting $\epsilon=\varepsilon r_{0} r \delta_{0}$, we would have

$$
\begin{gathered}
\left\|\mathrm{x}(t)-\mathrm{x}^{*}\right\| \leqslant \frac{r_{0}\left\|\mathrm{x}_{0}\right\|}{1+\|A\| t^{\alpha}}+\int_{0}^{t} \frac{\epsilon\|\mathrm{x}(t)\|}{1+\|A\| \tau^{\alpha}} \frac{(t-\tau)^{\alpha-1}}{\left(1+\|A\|(t-\tau)^{\alpha}\right)} \times \exp \left\{\int_{\tau}^{t} \frac{(t-s)^{\alpha-1}}{\left(1+\|A\|(t-s)^{\alpha}\right)} d s\right\} d \tau \\
\leqslant \frac{r_{0}\left\|\mathrm{x}_{0}\right\|}{1+\|A\| t^{\alpha}}+\int_{0}^{t} \frac{\epsilon\left\|\mathrm{x}_{0}\right\|(t-\tau)^{\alpha-1}}{\left(1+\|A\| \tau^{\alpha}\right)\left(1+\|A\|(t-\tau)^{\alpha}\right)^{1-1 / \alpha\|A\|}} d \tau,
\end{gathered}
$$

The integral equals to the sum of the two parts

$$
\begin{gathered}
\int_{0}^{t} \frac{\epsilon\left\|\mathrm{x}_{0}\right\|(t-\tau)^{\alpha-1}}{\left(1+\|A\| \tau^{\alpha}\right)\left(1+\|A\|(t-\tau)^{\alpha}\right)^{1-1 / \alpha\|A\|}} d \tau=\int_{0}^{t / 2} \frac{\epsilon\left\|\mathrm{x}_{0}\right\|(t-\tau)^{\alpha-1}}{\left(1+\|A\| \tau^{\alpha}\right)\left(1+\|A\|(t-\tau)^{\alpha}\right)^{1-1 / \alpha\|A\|}} d \tau \\
+\int_{t / 2}^{t} \frac{\epsilon\left\|\mathrm{x}_{0}\right\|(t-\tau)^{\alpha-1}}{\left(1+\|A\| \tau^{\alpha}\right)\left(1+\|A\|(t-\tau)^{\alpha}\right)^{1-1 / \alpha\|A\|}} d \tau
\end{gathered}
$$

Since $\alpha<1$ and $(t-\tau) \geqslant \tau$ when $\tau \in[0, t / 2]$, we would have

$$
\int_{0}^{t / 2} \frac{\epsilon\left\|\mathrm{x}_{0}\right\|(t-\tau)^{\alpha-1}}{\left(1+\|A\|(t-\tau)^{\alpha}\right)\left(1+\|A\| \tau^{\alpha}\right)^{1-1 / \alpha\|A\|}} d \tau \leqslant \int_{0}^{t / 2} \frac{\epsilon\left\|\mathrm{x}_{0}\right\|(\tau)^{\alpha-1}}{\left(1+\|A\| \tau^{\alpha}\right)\left(1+\|A\| \tau^{\alpha}\right)^{1-1 / \alpha\|A\|}} d \tau
$$


Similarly

$$
\begin{gathered}
\int_{t / 2}^{t} \frac{\epsilon\left\|\mathrm{x}_{0}\right\|(t-\tau)^{\alpha-1}}{\left(1+\|A\| \tau^{\alpha}\right)\left(1+\|A\|(t-\tau)^{\alpha}\right)^{1-1 / \alpha\|A\|}} d \tau \leqslant \int_{t / 2}^{t} \frac{\epsilon\left\|\mathrm{x}_{0}\right\|(t-\tau)^{\alpha-1}}{\left(1+\|A\|(t-\tau)^{\alpha}\right)\left(1+\|A\|(t-\tau)^{\alpha}\right)^{1-1 / \alpha\|A\|}} d \tau \\
=\int_{t / 2}^{t} \frac{\epsilon\left\|\mathrm{x}_{0}\right\| s^{\alpha-1}}{\left(1+\|A\| s^{\alpha}\right)\left(1+\|A\| s^{\alpha}\right)^{1-1 / \alpha\|A\|}} d s
\end{gathered}
$$

By substituting $s$ for $(t-\tau)$ and by considering $\left|\arg \left(\lambda_{i}\right)\right|>\alpha \frac{\pi}{2}$ we would have $\alpha\|A\|>1$, resulting in the following inequality

$$
\begin{gathered}
\left\|\mathrm{x}(t)-\mathrm{x}^{*}\right\| \leqslant \frac{r_{0}\left\|\mathrm{x}_{0}\right\|}{1+\|A\| t^{\alpha}}+2 \int_{0}^{t / 2} \frac{\epsilon\left\|\mathrm{x}_{0}\right\|}{\left(1+\|A\| \tau^{\alpha}\right)^{(2-1 / \alpha\|A\|)}} d \tau, \\
=\frac{r_{0}\left\|\mathrm{x}_{0}\right\|}{1+\|A\| t^{\alpha}}+\frac{2 \epsilon\left\|\mathrm{x}_{0}\right\|}{\alpha\|A\|-1}+\frac{2 \epsilon\left\|\mathrm{x}_{0}\right\|}{(1-\alpha\|A\|)\left(1+\|A\|\left(\frac{t}{2}\right)^{\alpha}\right)^{1-1 / \alpha\|A\|}}
\end{gathered}
$$

Since $\delta$ and $\delta_{0}$ are arbitrarily small, we would have

$$
\lim _{t \rightarrow \infty}\left(\frac{r_{0}\left\|\mathrm{x}_{0}\right\|}{1+\|A\| t^{\alpha}}+\frac{2 \epsilon\left\|\mathrm{x}_{0}\right\|}{\alpha\|A\|-1}+\frac{2 \epsilon\left\|\mathrm{x}_{0}\right\|}{(1-\alpha\|A\|)\left(1+\|A\|\left(\frac{t}{2}\right)^{\alpha}\right)^{1-1 / \alpha\|A\|}}\right)=0
$$

Therefore, the solution of system (8) is asymptotically stable.

Remark 2.1. Let $B=\operatorname{bcirc}(A, C, \ldots, C) \in \mathbb{C}^{N \times N}$ then

$$
\lambda(B)=\{\lambda(A+(N-1) C), \underbrace{\lambda(A-C), \ldots, \lambda(A-C)}_{N-1}\}
$$

\section{Stability of FMHNN Model}

Theorem 2.4. The system (1) is asymptotically stable at its equilibrium point, $E^{\star}=[0,0, \delta]$, if and only if one of the following conditions hold

$$
\begin{aligned}
& i . \eta>0 \text { and } \frac{\tau}{\sqrt{\eta}}<2 \cos \left(\frac{\alpha \pi}{2}\right) \\
& \text { ii . } \eta=0 \text { and } \delta=-3 / k
\end{aligned}
$$

where $\eta=\left(b_{1}+k \delta-1\right)\left(b_{4}-k \delta-1\right)-\left(b_{3}-k \delta\right)\left(b_{2}-k \delta\right)$ and $\tau=b_{1}+b_{4}+2(k \delta-1)$.

Proof. The Jacobian matrix of the system (1) is as follow

$$
J\left[x_{1}, x_{2}, \varphi\right]=\left[\begin{array}{ccc}
-1+b_{1} \operatorname{sech}^{2}\left(x_{1}\right)+k \varphi & b_{2} \operatorname{sech}^{2}\left(x_{2}\right)-k \varphi & k\left(x_{1}-x_{2}\right) \\
b_{3} \operatorname{sech}^{2}\left(x_{1}\right)-k \varphi & -1+b_{4} \operatorname{sech}^{2}\left(x_{2}\right)+k \varphi & -k\left(x_{1}-x_{2}\right) \\
1 & -1 & 0
\end{array}\right]
$$

which at its equilibrium $E^{\star}=[0,0, \delta]$ will be

$$
J\left(E^{\star}\right)=\left[\begin{array}{ccc}
-1+b_{1}+k \delta & b_{2}-k \delta & 0 \\
b_{3}-k \delta & -1+b_{4}+k \delta & 0 \\
1 & -1 & 0
\end{array}\right]
$$


Thus, characteristic equation of matrix (10) will be in the form of $p(\lambda)=\lambda p_{1}(\lambda)$ where

$$
p_{1}(\lambda)=\lambda^{2}+\left[-b_{1}-b_{4}+2(1-k \delta)\right] \lambda+\left(1-b_{1}\right)\left(1-b_{4}\right)-b_{2} b_{3}+\left(b_{1}+b_{2}+b_{3}+b_{4}-2\right) k \delta
$$

Assuming $\tau=-b_{1}-b_{4}+2(1-k \delta)$ and $\eta=\left(1-b_{1}\right)\left(1-b_{4}\right)-b_{2} b_{3}+\left(b_{1}+b_{2}+b_{3}+b_{4}-2\right) k \delta$, we can re-write the characteristic equation in the form of

$$
p(\lambda)=\lambda\left(\lambda^{2}-\tau \lambda+\eta\right)
$$

according to lemma (2.2) then, the proof of (i) would be straightforward. For case (ii), when $\eta=0$, then $\delta=-3 / k$ and the eigenvalues $\lambda_{1,2}$ are written as $\lambda_{1}=0, \lambda_{2}=1.9+2 k \delta=-4.1$, resulting in a negative real root and a zero root. Based on lemma (2.1) therefore, $E^{\star}$ would be a stable point (Table. 1).

Remark 2.2. When $\eta<0$ then $\tau^{2}-4 \eta>0$ and $\delta>1.9512 / k$ or $\delta<-3 / k$. Therefore, $\lambda_{1,2}$ has at least one positive real root, which shows $E^{\star}$ is an unstable point.

\section{Stability of FMHNN Model with Ring Structure}

Theorem 2.5. The system (3) is locally asymptotically stable at its equilibrium point, $\mathrm{X}^{\star}=\left(0,0, \phi^{\star}\right)$, when $1<n<(2 p(d+1)+d) / d$ and $0<\alpha<1$.

Proof. First we re-write the system (3) in compact form as below

$$
\mathbb{F}(X)=A X+\mathbb{H}(X)
$$

where $\mathbb{H}(X)=B_{1} \tanh \left(\mathrm{x}_{1}\right)+B_{2} \tanh \left(\mathrm{x}_{2}\right)+\varphi K X, A=\operatorname{bcirc}\left(A_{0}, A_{1}, \ldots, A_{1}\right) \in \mathbb{R}^{n \times n}, B_{1}=\left[\beta_{1}\right]_{1 \times n}, B_{2}=$ $\left[\beta_{2}\right]_{1 \times n}, \beta_{1}=\left[b_{1}, b_{3}, 0\right], \beta_{2}=\left[b_{2}, b_{4}, 0\right]$, and $K=\operatorname{bcirc}(\kappa, 0, . ., 0)_{1 \times n}$ so that $A_{0}, A_{1} \in \mathbb{R}^{3 \times 3}$ and

$$
A_{0}=\left[\begin{array}{ccc}
-1-\frac{d}{p} & 0 & 0 \\
0 & -1 & 0 \\
1 & -1 & 0
\end{array}\right], \quad A_{1}=\left[\begin{array}{ccc}
\frac{d}{2 p} & 0 & 0 \\
0 & 0 & 0 \\
0 & 0 & 0
\end{array}\right], \quad \kappa=\left[\begin{array}{ccc}
-k & k & 0 \\
k & -k & 0 \\
0 & 0 & 0
\end{array}\right]
$$

Next we show that equation (12) satisfies stability condition at its equilibrium point, $X^{\star}=\left(0,0, \phi^{\star}\right)$ because

$$
\lim _{X \rightarrow X^{*}} \frac{\|\mathbb{H}(X)\|}{\left\|X-X^{\star}\right\|}=\lim _{X \rightarrow X^{*}} \frac{\left(\left\|B_{1}\right\|\left\|\tanh \left(\mathrm{x}_{1}\right)\right\|+\left\|B_{2}\right\|\left\|\tanh \left(\mathrm{x}_{2}\right)\right\|+\|\varphi\|\|K\|\|X\|\right)}{\left\|X-X^{\star}\right\|}=0
$$

which this satisfies one of conditions of theorem (2.2.2). The other condition also holds when $1<n<$ $(2 p(d+1)+d) / d$ and $0<\alpha<1$ because

$$
\begin{aligned}
\operatorname{det}(A-\lambda I) & =\left\{\lambda\left(A_{0}+(n-1) \times A_{1}\right), \lambda\left(A_{0}-A_{1}\right) \times(n-1)\right\} \\
& =\left\{-1,0, \frac{d(n-1)-2 p(d+1)}{2 p}, 1-n, 0,(1-n)(3 d / 2 p+1)\right\}
\end{aligned}
$$

Remark 2.3. For $\alpha=1$, under the assumption of $1<n<(2 p(d+1)+d) / d$ at equilibrium point, $\mathrm{X}^{\star}=\left(0,0, \phi^{\star}\right)$, the system (3) would have limit cycles. 
Table 1: Stability region of two-neuron FMHNN model (1) under $b_{1}=-0.1, b_{2}=2.8, b_{3}=-3$, and $b_{4}=4$ assumption.

\begin{tabular}{lccc}
\hline Condition on $\eta$ & Condition on $\tau, \delta$ & Stability conditions & Stability Cases \\
\hline$\eta=0$ & $\delta=-3 / k$ & Stable & Case 1 \\
$\eta>0$ & $\tau=0, \delta=-0.95 / k$ & Stable & Case 2 \\
$\eta>0$ & $\tau>0,-0.95 / k<\delta<1.9512 / k$ & Stable & Case 3 \\
$\eta>0, \tau^{2}<4 \eta$ & $\tau<0,-0.95 / k<\delta<1.9512 / k$ & $\cos \left(\frac{\alpha \pi}{2}\right)>\frac{\tau}{2 \sqrt{\eta}}$ & Case 4 \\
$\eta<0$, & $\delta>1.9512 / k$ oR $\delta<-3 / k$ & Unstable & Case 5 \\
\hline
\end{tabular}

Table 2: The amount of eigenvalues for the stability region of model (1) according table (1) when $k=0.15$.

\begin{tabular}{lllc}
\hline \multicolumn{1}{c}{$\delta$} & Equilibrium $\left(E^{\star}\right)$ & Eigenvalues $\lambda_{1,2}$ & Stability Cases \\
\hline-20 & $(0,0,-20)$ & $0,-4.1000$ & Case 1 \\
-6.3333 & $(0,0,-6.3333)$ & $\pm 1.8668 i$ & Case 2 \\
-18 & $(0,0,-18)$ & $-3.3477,-0.1523$ & Case 3 \\
-8 & $(0,0,-6)$ & $-0.2500 \pm 1.7313 i$ & Case 3 \\
-5 & $(0,0,-5)$ & $0.2 \pm 1.9455 i$ & Case 4 \\
10 & $(0,0,10)$ & $2.4500 \pm 1.2835 i$ & Case 4 \\
14 & $(0,0,14)$ & $3.8453,2.2547$ & Case 5 \\
-25 & $(0,0,-25)$ & $0.2191,-5.8191$ & Case 5 \\
\hline
\end{tabular}

\section{Results}

In this section, we numerically scrutinize our analytical analyses for both systems (1 \& 3) using FDE12 package in Matlab [19]. We also examine the influence of the memory element, $\alpha$, on the bifurcation patterns.

FMHNN Model. We set $b_{1}=-0.1, b_{2}=2.8, b_{3}=-3$ and $b_{4}=4$ in system (1), as assumed by Chen et al [4]. Based on equation (11) therefore, at the equilibrium point, $E^{\star}=(0,0, \delta)$, we would have the following characteristic equation for system (1)

$$
P(\lambda)=\lambda\left[\lambda^{2}-(1.9+2 k \delta) \lambda+5.1+1.7 k \delta\right]
$$

Considering $\tau=-(1.9+2 k \delta), \eta=5.1+1.7 k \delta$, and using theorem (2.4), we can summarize the stability conditions of our two-neuron fmHNN model (1) in table (1). Next, we consider $k=0.15$ and by this way, we examine the stability criteria of our system over upper and lower bound of $\delta$ value (table 2).

Based on table (2), the system (1) is locally asymptotically stable in the first three regions since lemma (2.1) holds for these cases. With regard to region 4 , we have $-6.3<\delta<13$, so we examine two equilibrium point $\left(\delta=-5\right.$ and $\delta=10$ ). According to the amount of eigenvalues $\left(\lambda_{1,2}\right)$, given in table (2), at the equilibrium points $E_{1}^{\star}=(0,0,-5)$ and $E_{2}^{\star}=(0,0,10)$, the stability condition satisfies when $\alpha<0.9348$ and $\alpha<0.307$ respectively (Fig. 3).

As for region 5, we examine two equilibrium points $(\delta=14$ and $\delta=-25)$. Based on the amount of eigenvalues $\left(\lambda_{1,2}\right)$, given in table (2), our system is not stable in any of these two cases because the stability criteria is violated, having positive real roots for the characteristic equation (Fig. 4).

FMHNN Model with Ring Structure. In addition to our assumption about the parameters FMHNN model, here we also set $p=1$, and $d=0.5$. According to the compact for of model (12) then, we would 

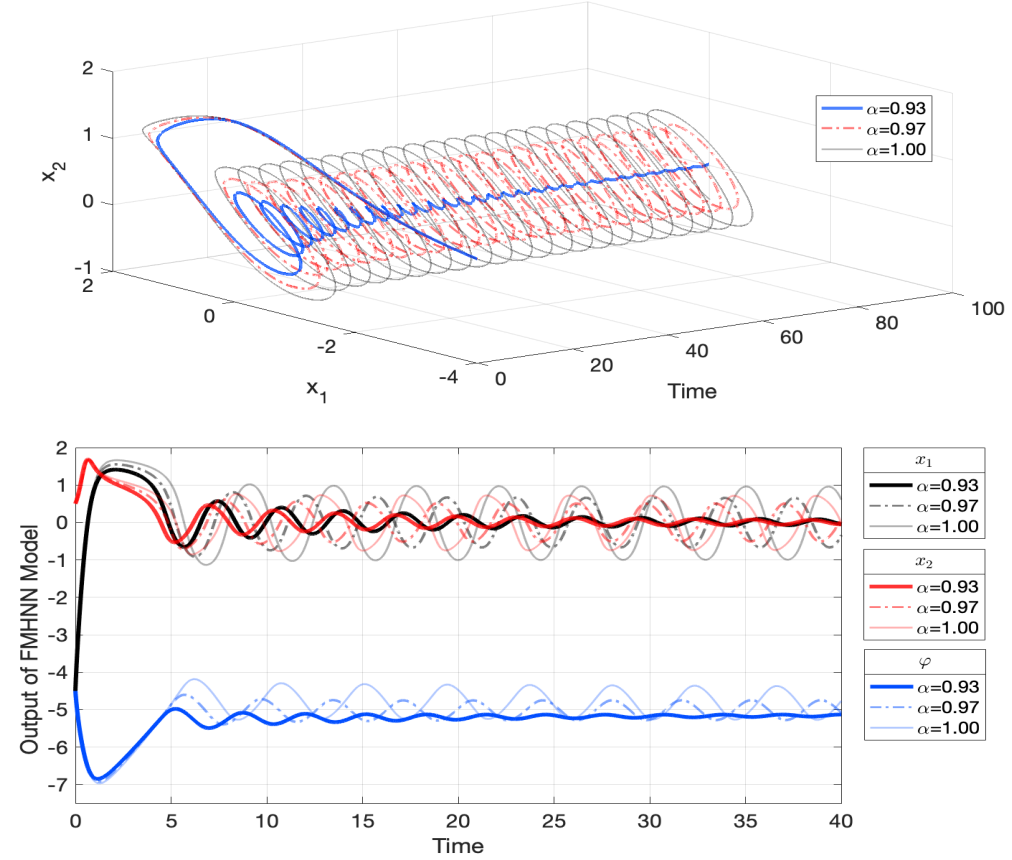

Figure 3: Phase diagrams and time series of FMHNN model (1) in case $4, \delta=-5$, with initial value $\left(x_{1}(0), x_{2}(0), \varphi(0)\right)=$ $(-4.5,0.5,-4.5)$ for three different fractional orders. Details are given in the legends.
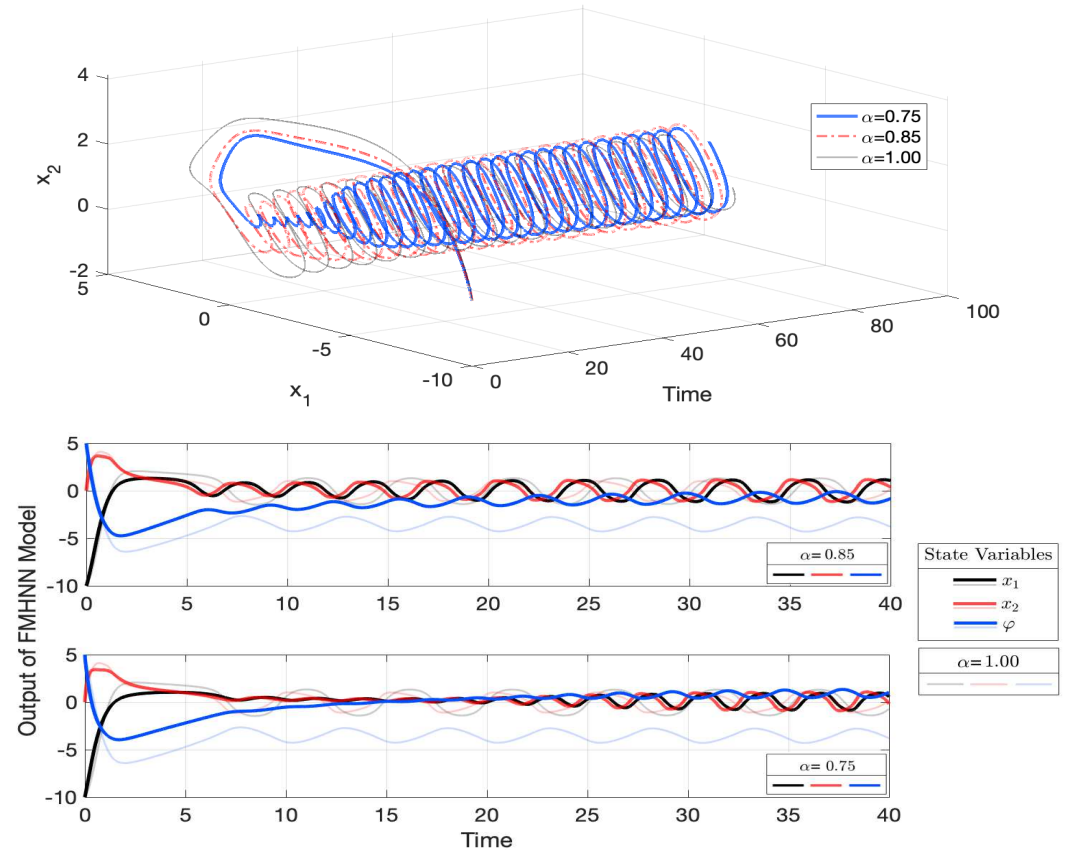

Figure 4: Phase diagrams and time series of FMHNN model (1) in case $5, \delta=14$, with initial value $\left(x_{1}(0), x_{2}(0), \varphi(0)\right)=\left(-10,10^{-6}, 5\right)$ for three different fractional orders. Details are given in the legends. 
have $\beta_{1}=[-0.1,-3,0], \beta_{2}=[2.8,4,0]$ and

$$
A_{0}=\left[\begin{array}{ccc}
-1.1 & 0 & 0 \\
0 & -1 & 0 \\
1 & -1 & 0
\end{array}\right], A_{1}=\left[\begin{array}{ccc}
0.05 & 0 & 0 \\
0 & 0 & 0 \\
0 & 0 & 0
\end{array}\right], \kappa=\left[\begin{array}{ccc}
-0.15 & 0.15 & 0 \\
0.15 & -0.15 & 0 \\
0 & 0 & 0
\end{array}\right]
$$

which satisfies the stability criteria represented by theorem (2.5) when

$$
1<n<(2(1)(1+0.5)+0.5) / 0.5=7
$$

Therefore, under our parameter assumption when $n<7$, the model (3) would be stable for $0<\alpha<1$, and it would have stable limit cycles for $\alpha=1$ when $n<7$ (Fig. 5).

Bifurcation Analysis. Here we would study the stability criteria further through investigating the influences of the initial conditions and the fractional-order parameter $\alpha$ on our FMHNN model (1). Again, we set $b_{1}=-0.1, b_{2}=2.8, b_{3}=-3$ and $b_{4}=4$ in system (1), as assumed by Chen et al [4]. Taking $\alpha$ as the bifurcation parameter when $\alpha \in[0.96,1]$ and the initial value $\left[0,10^{-6}, 0\right]$, we performed the the bifurcation analysis. Figure (6) shows the bifurcation diagram of the $\alpha-\phi$ plane, indicating that with the increase of $\alpha$ value the trajectory of FMHNN evolves into unbounded behavior, chaos, eight-period cycles, four-period and finally to two-period cycles. Also according to the Lyapunov exponent shown in figure (6), the system can not be asymptotically stable for the given interval value of $\alpha$.

\section{Discussion}

Understanding the dynamics of fractional-order memristor synapse-coupled Hopfield neural network is crucial due to its application in designing complex systems in order to examine the behaviour of the brain, motivating us to investigate the impact of its parameters and the fractional calculus concept on outputs. Having said so, we first introduced a two-neuron network model to study the impact of the memory element, fractional-order $\alpha$, on the system. The motivation is to determine the influence of the memory concept on the chaos behaviour observed in the integer case by Chen et al [4]. Our analytical calculations and numerical simulations reveal that the memory element plays a pivotal role in the stability criteria. In effect, the fractionalized model could be stable over some $\alpha$ ranges, depending on the parameters. To compare the findings more closely with the integer case, under the same assumption as Chen et al [4], we performed bifurcation analysis and estimate Lyapunov exponents. The outputs indicate that the model is unstable when $\alpha=1$, and also experiences several dynamical transitions from unbounded to non-chaotic behaviour by increasing $\alpha$ value from 0.96 to 1 . For the value less than 0.9348 also, the fractional system is stable.

Next, we expand our model to a general fractional-order network using the ring concept, where each membrane is coupled by $n$ sub-networks. The stability criteria are derived, showing the dependency of the conditions on the value of $n$. That is, the number of sub-networks, $n$, in membranes is of vital importance in stabilizing the system's behaviour. For $\alpha$ is less than 1 as well, the model converges to the asymptotically stable solution, whilst for $\alpha=1$, it leads to limit cycles.

It should also be mentioned that based on the numerical simulation, the trajectory behaviour of both models dampens out induced oscillating inherent and reaches the local stable point quicker with decreasing $\alpha$ value. As future work, we will study how incorporating the delay concept changes the stability criteria and the findings.

\section{Data availability statement}

No data were used to support this study. 

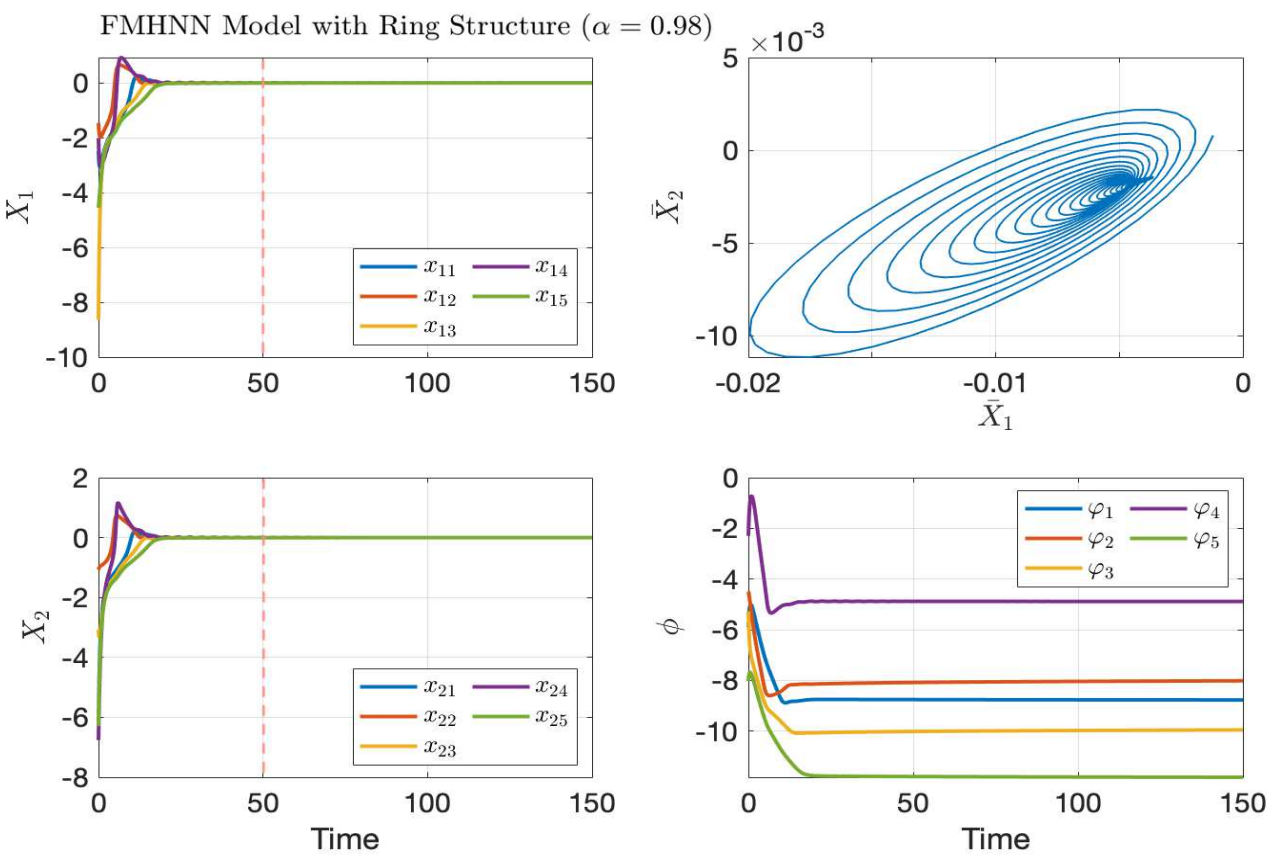

FMHNN Model with Ring Structure $(\alpha=1.00)$
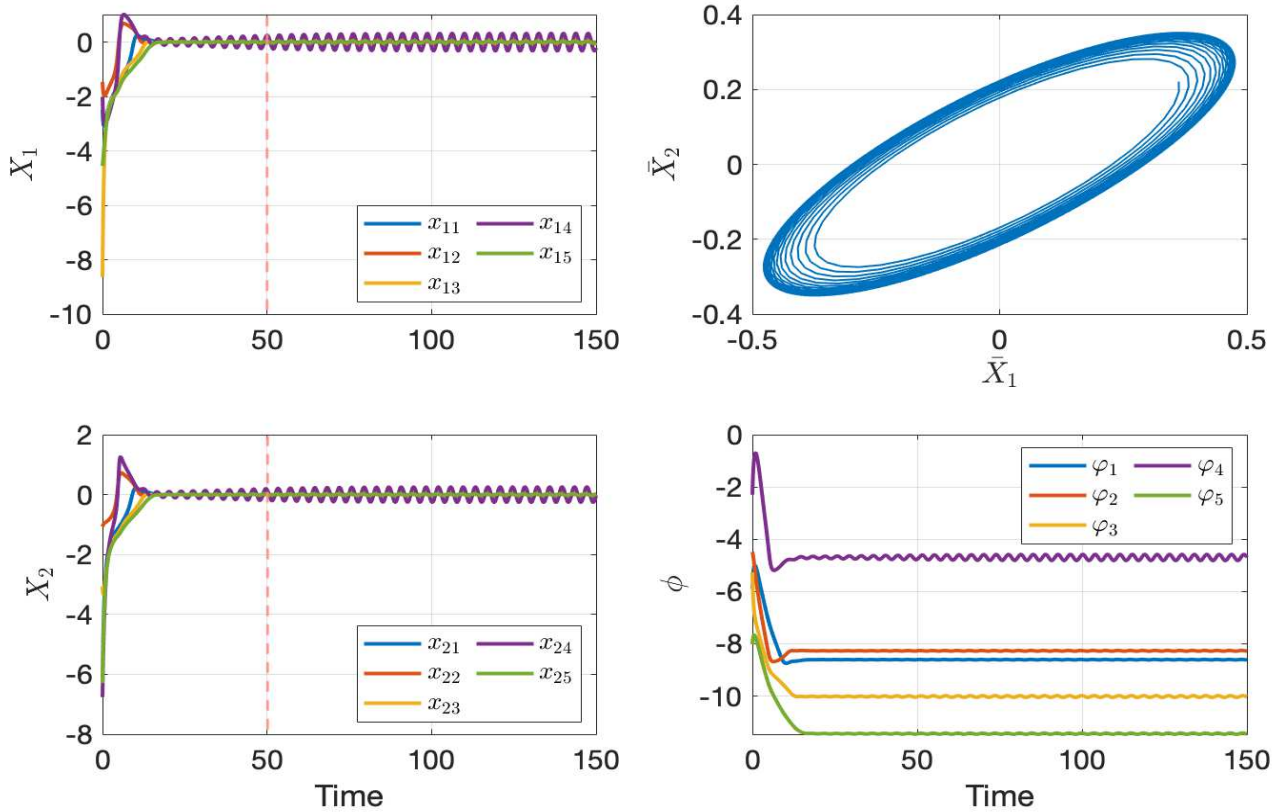

Figure 5: Phase diagrams and time series of FMHNN model with ring structure (3) consisting of 5 sub-networks with $p=1$, $d=0.5$ and initial condition $X_{1}=(-2.48,-6.12,-5.90,-1.46,-1.07), X_{2}=(-4.48,-8.64,-3.06,-5.27,-2.01), \phi=$ $(-6.76,-2.30,-4.55,-6.30,-8.02)$ where $X_{1}=\left[x_{1 i}\right], X_{2}=\left[x_{2 i}\right], \phi=\left[\varphi_{i}\right]$ for $i=1, \cdots, 5 . \bar{X}_{1}=\frac{1}{5} \sum_{i=1}^{5} x_{1 i}$, and $\bar{X}_{2}=\frac{1}{5} \sum_{i=1}^{5} x_{2 i}$ are average potentials between two neurons, calculated for $t \geq 50$. Further details are given in the titles and legends. 

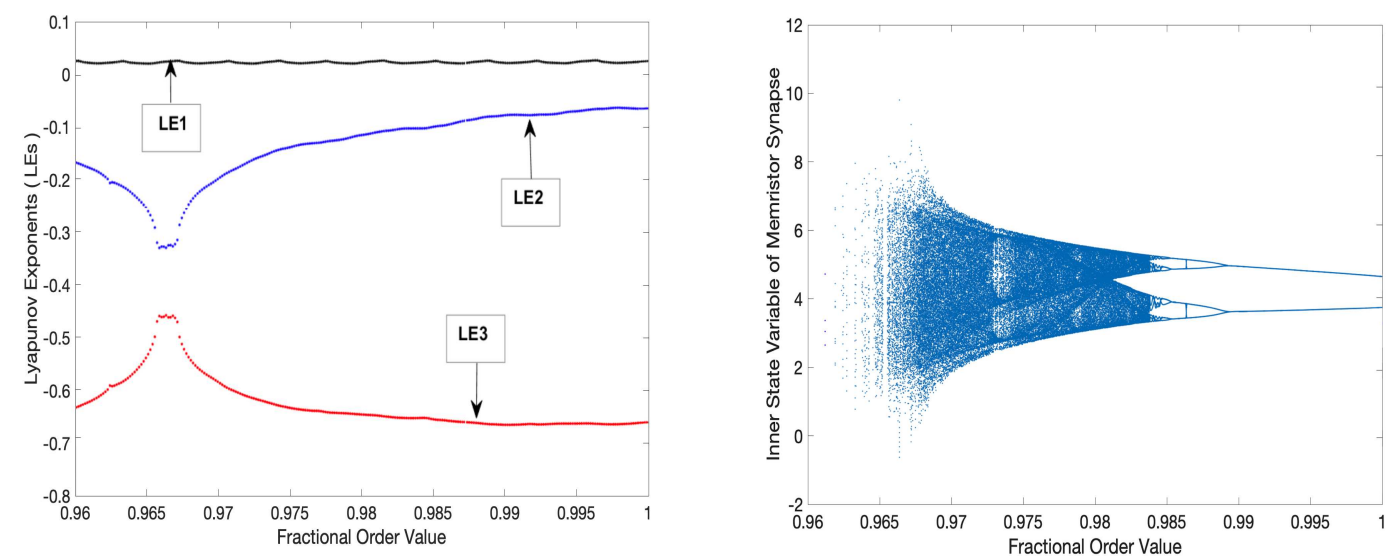

Figure 6: The bifurcation diagram and Lyapunov exponents of FMHNN model (1) on the $\alpha-\phi$ plane over $0.96 \leq \alpha \leq 1$ interval.

\section{Statements and Declarations}

The authors did not receive support from any organization for the submitted work. Also they declare that they have no known competing financial interests or personal relationships that could have appeared to influence the work reported in this paper.

\section{References}

[1] Qingdu Li and Xiaosong Yang. "Complex dynamics in a simple Hopfield-type neural network". In: International Symposium on Neural Networks. Springer. 2005, pp. 357-362.

[2] S He. "Complexity and Chimera States in a Ring-Coupled Fractional-Order Memristor Neural Network". In: Front. Appl. Math. Stat. 6: 24. doi: 10.3389/fams (2020).

[3] ZT Njitacke, J Kengne, and HB Fotsin. "A plethora of behaviors in a memristor based Hopfield neural networks (HNNs)". In: International Journal of Dynamics and Control 7.1 (2019), pp. 3652.

[4] Jiejie Chen, Zhigang Zeng, and Ping Jiang. "Global Mittag-Leffler stability and synchronization of memristor-based fractional-order neural networks”. In: Neural Networks 51 (2014), pp. 1-8.

[5] CJ Zuñiga Aguilar et al. "Fractional order neural networks for system identification". In: Chaos, Solitons $\mathcal{E}$ Fractals 130 (2020), p. 109444.

[6] Mohammad Amirian Matlob and Yousef Jamali. "The concepts and applications of fractional order differential calculus in modeling of viscoelastic systems: a primer". In: Critical Reviews ${ }^{\mathrm{TM}}$ in Biomedical Engineering 47.4 (2019).

[7] HongGuang Sun et al. "A new collection of real world applications of fractional calculus in science and engineering". In: Communications in Nonlinear Science and Numerical Simulation 64 (2018), pp. 213-231.

[8] Mohammad M Amirian et al. "Memory and mutualism in species sustainability: A time-fractional Lotka-Volterra model with harvesting". In: Heliyon 6.9 (2020), e04816.

[9] M Saeedian et al. "Memory effects on epidemic evolution: The susceptible-infected-recovered epidemic model”. In: Physical Review E 95.2 (2017), p. 022409. 
[10] Moein Khalighi et al. "Three-species Lotka-Volterra model with respect to Caputo and CaputoFabrizio fractional operators”. In: Symmetry 13.3 (2021), p. 368.

[11] Igor Podlubny. Fractional differential equations: an introduction to fractional derivatives, fractional differential equations, to methods of their solution and some of their applications. Elsevier, 1998.

[12] Chengjie Chen et al. "Coexisting multi-stable patterns in memristor synapse-coupled Hopfield neural network with two neurons”. In: Nonlinear Dynamics 95.4 (2019), pp. 3385-3399.

[13] Moein Khalighi, Mohammad Amirianmatlob, and Alaeddin Malek. "A new approach to solving multiorder time-fractional advection-diffusion-reaction equations using BEM and Chebyshev matrix”. In: Mathematical Methods in the Applied Sciences 44.4 (2021), pp. 2964-2984.

[14] Ali Kaveh and Hossein Rahami. "Block circulant matrices and applications in free vibration analysis of cyclically repetitive structures". In: Acta Mechanica 217.1 (2011), pp. 51-62.

[15] Garry J Tee. "Eigenvectors of block circulant and alternating circulant matrices". In: New Zealand Journal of Mathematics 36.8 (2007), pp. 195-211.

[16] Denis Matignon. "Stability results for fractional differential equations with applications to control processing”. In: Computational engineering in systems applications. Vol. 2. 1. Lille, France. 1996, pp. 963-968.

[17] Eva Kaslik and Seenith Sivasundaram. "Nonlinear dynamics and chaos in fractional-order neural networks". In: Neural Networks 32 (2012), pp. 245-256.

[18] Xiang-Jun Wen, Zheng-Mao Wu, and Jun-Guo Lu. "Stability analysis of a class of nonlinear fractional-order systems". In: IEEE Transactions on circuits and systems II: Express Briefs 55.11 (2008), pp. 1178-1182.

[19] Roberto Garrappa. "Numerical solution of fractional differential equations: A survey and a software tutorial”. In: Mathematics 6.2 (2018), p. 16. 